\title{
Targeting HDAC with a novel inhibitor effectively reverses paclitaxel resistance in non-small cell lung cancer via multiple mechanisms
}

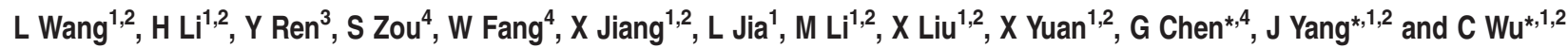

Chemotherapy paclitaxel yields significant reductions in tumor burden in the majority of advanced non-small cell lung cancer (NSCLC) patients. However, acquired resistance limits its clinical use. Here we demonstrated that the histone deacetylase (HDAC) was activated in paclitaxel-resistant NSCLC cells, and its activation promoted proliferation and tumorigenesis of paclitaxelresistant NSCLC cells in vitro and in vivo. By contrast, knockdown of HDAC1, a primary isoform of HDAC, sensitized resistant cells to paclitaxel in vitro. Furthermore, we observed that overexpression of HDAC1 was associated with the downregulation of p21, a known HDAC target, in advanced NSCLC patients with paclitaxel treatment, and predicted chemotherapy resistance and bad outcome. In addition, we also identified a novel HDACs inhibitor, SNOH-3, which inhibited HDAC expression and activity, induced cell apoptosis, and suppressed cell migration, invasion and angiogenesis. Notably, co-treatment with SNOH-3 and paclitaxel overcome paclitaxel resistance through inhibiting HDAC activity, leading to the induction of apoptosis and suppression of angiogenesis in vitro and in preclinical model. In summary, our data demonstrate a role of HDAC in paclitaxel-resistant NSCLC and provide a promising therapeutic strategy to overcome paclitaxel-acquired resistance.

Cell Death and Disease (2016) 7, e2063; doi:10.1038/cddis.2015.328; published online 21 January 2016

Non-small cell lung cancer (NSCLC) is one of the main cause of cancer-related death worldwide. ${ }^{1}$ Standard therapeutic approaches for NSCLC include surgical resection, chemoand radio-therapy. ${ }^{2}$ Due to advanced disease at diagnosis, a majority of patients are not candidates for surgery. Therefore, chemotherapeutic approaches are irreplaceable for advanced NSCLC. ${ }^{3}$ Paclitaxel is a taxane antimitotic agent currently used as the first-line standard therapy regimen with platinum agent for advanced NSCLC. ${ }^{4-6}$ In addition, paclitaxel as a single agent has also shown efficacy in the second-line setting in advanced and metastatic NSCLC. ${ }^{5,6}$ However, acquired resistance inexorably develops after a period of treatment. ${ }^{7}$ Paclitaxel resistance can be achieved through several mechanisms, including tubulin isoforms/mutations and the alteration of drug efflux pumps. ${ }^{7,8}$ Other mechanisms of resistance have also been identified, including deregulation of apoptotic signaling pathways and activation of hypoxiainduced factor 1 (HIF-1) signaling..$^{9-11}$ Unfortunately, in spite of these advances, treatment of paclitaxel-resistant patients remains a critical clinical challenge. Thus, there is an intense need to further understand mechanisms of paclitaxel resistance.

Epigenetic changes, which are somatically inherited through cell division, are considered as the potential drivers of drug resistance in cancer. ${ }^{12}$ It has been demonstrated that the high rate of epigenetic change in tumors generates diversity in gene expression patterns that can rapidly evolve through drug selection during treatment, leading to the development of acquired resistance. ${ }^{12}$ Histone acetylation, which is the result of the balance between the activity of histone deacetylases (HDACs) and histone acetyltransferases (HATs), is recognized as an important epigenetic event. $^{13}$ The acetylation of histones in nucleosomes contributes to changes in chromatin conformation and mediates to regulation of gene expression. ${ }^{14} \mathrm{~A}$ lot of studies verified that the aberrantly overexpressed HDACs in various tumors leading to carcinogenesis, cancer progression, and clinical poor outcome. ${ }^{15-17}$ Thus, HDACs can be a therapeutic intervention for cancer treatment to reverse aberrant epigenetic states associated with cancer, especially drug resistance. ${ }^{12,18}$ In fact, several studies have illustrated that the synergistic therapeutic effects have been obtained from HDAC inhibitors in combination with DNA-damaging agents, taxanes, targeted agents, death receptor agonists, and hormonal therapies. ${ }^{18}$ Furthermore, HDAC inhibitors have also been found to reverse cytotoxic and targeted agent resistance in various tumors. ${ }^{18-20}$ However, whether HDACs are implicated in paclitaxel resistance in NSCLCs is still not elucidated. Recently, a clinical study showed that vorinostat, an FDA-approved HDACs inhibitor, enhanced the efficacy of paclitaxel combined with carboplatin in patients with

\footnotetext{
${ }^{1}$ Department of Pharmacology, Shenyang Pharmaceutical University, Shenyang, PR China; ${ }^{2}$ Benxi Institute of Pharmaceutical Research, Shenyang Pharmaceutical University, Shenyang, PR China; ${ }^{3}$ Department of Pathology, Wuhan General Hospital of Guangzhou Command, People's Liberation Army, Wuhan, PR China and ${ }^{4}$ Key Laboratory of Structure-Based Drugs Design and Discovery of Ministry of Education, Shenyang Pharmaceutical University, Shenyang, PR China

${ }^{*}$ Corresponding author: G Chen, Key Laboratory of Structure-Based Drugs Design and Discovery of Ministry of Education, Shenyang Pharmaceutical University, Shenyang, PR China. Tel: +86 24 23986412; Fax: +86 24 31306459; E-mail: guoliang222@gmail.com or J Yang or C Wu, Department of Pharmacology, Shenyang Pharmaceutical University, Shenyang 110016, PR China. Tel: +86 24 23986011; Fax: +86 24 23986339; E-mail: yangjingyu2006@gmail.com or wucf@syphu.edu.cn

Abbreviations: NSCLC, non-small cell lung cancer; HDAC, histone deacetylase; Ac-H3, acetylated Histone 3; Ac-H4, acetylated Histone 4; TTP, time to tumor progression; OS, overall survival; A549/T, paclitaxel-resistant A549; HUVECs, human umbilical vein endothelial cells; Cl, combination index; MVD, microvessel density Received 22.8.15; revised 25.9.15; accepted 06.10.15; Edited by R Johnstone
} 
advanced NSCLC, ${ }^{21}$ suggesting that HDACs might have an important role in paclitaxel resistance.

Here, we disclosed that paclitaxel-resistant NSCLC cells displayed the enhanced HDAC activity, increased HDAC1 expression, and raised cell proliferation ability in vitro and in vivo, and HDAC1 knockdown could sensitize resistant cells to paclitaxel in vitro. Clinically, patients owing higher HDAC1 with lower p21 expression tumors showed chemotherapy resistance and bad outcome while taking paclitaxel as a chemotherapy agent. In addition, we also identified a novel HDACs inhibitor, SNOH-3, which was more potent than FDAapproved HDAC inhibitor SAHA to inhibit HDAC activity, induce cell apoptosis, and suppress cell migration, invasion and angiogenesis. Furthermore, when SNOH-3 combined with paclitaxel could overcome paclitaxel resistance through inhibiting HDAC activity, leading to the induction of apoptosis and suppression of angiogenesis. Our results not only identify a therapeutic strategy to abrogate acquired resistance to paclitaxel in NSCLC, but also imply that HDAC1 along with p21 might be a predictive biomarker for paclitaxel treatment.

\section{Results}

Paclitaxel-resistant NSCLC cells showed enhanced HDAC activity and tumorigenicity. To study the phenomenon of relapse after paclitaxel therapy, we generated in vitro chemoresistant model using the NSCLC cell line A549. Drugresistant cells were established by exposure to increasing concentrations of paclitaxel, and resistance was validated by cell viability. As shown in Figure 1a, as compared with parental cells, A549/T cells exhibited an increased resistance to paclitaxel, with the resistance index is 6.19 . In view of the

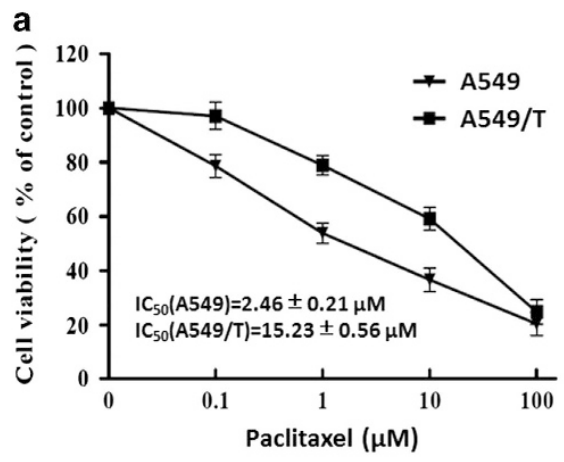

b
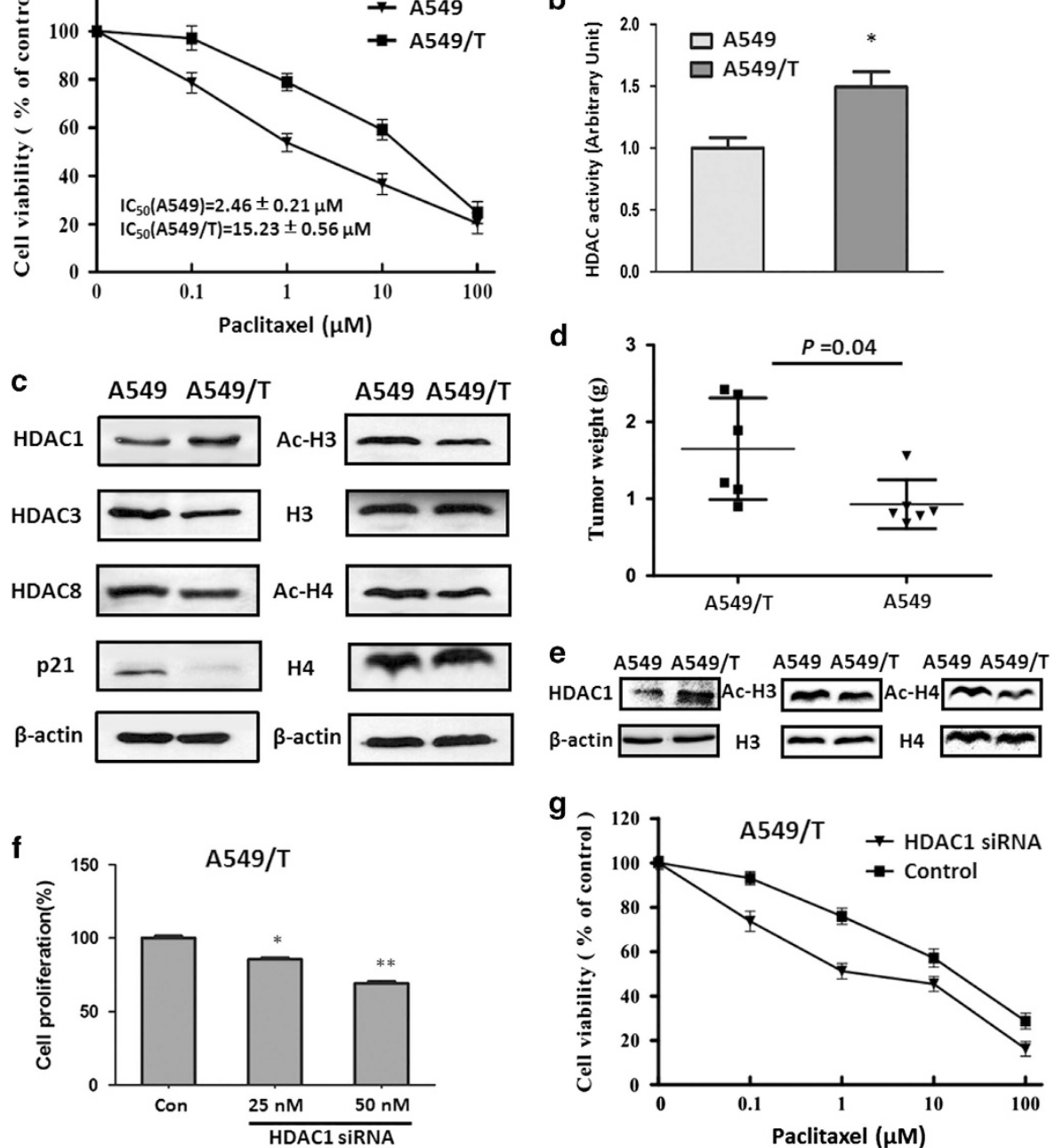

Figure 1 HDAC activity and function in paclitaxel-resistant NSCLC cells. (a) The effect of paclitaxel on the cell viability of A549 and A549/T cells. The cells were treated with various concentrations of paclitaxel for $48 \mathrm{~h}$. (b) The HDAC activity in A549 and A549/T cells. (c) HDAC1, HDAC3, HDAC8, acetylated Histone-3 (Ac-H3), Histone-3(H3), acetylated Hstone-4 (Ac-H4), Histone-4 (H4), and p21 were measured in A549 and A549/T cell lines. $\beta$-Actin expression was used as a loading control. (d) The tumor weight of A549 and A549/T xenograft mouse. (e) HDAC1, Ac- H3, H3, Ac-H4, and $\mathrm{H} 4$ were measured in A549 and A549/T tumor tissues. $\beta$-Actin expression was used as a loading control. (f) The proliferation rate of A549/T cells after treated with different concentrations of HDAC siRNA ( 25 and $50 \mathrm{nM}$ ) for $48 \mathrm{~h}$. (g) The effect of knockdown of HDAC1 by siRNA $(50 \mathrm{nM})$ on the sensitivity to paclitaxel in A549/T cells. ${ }^{*} P<0.05,{ }^{* *} P<0.01$ compare with control 
crucial role of HDAC in drug resistance of various tumors, ${ }^{12}$ we also detected the HDAC activity of A549/T and its parental cells. Our results indicated that HDAC activity was increased to 1.5-fold in A549/T cells when compared with that of parental A549 cells (see Figure 1b). Furthermore, we also measured the expression levels of HDACs isoforms, including HDAC1, HDAC3, and HDAC8, in this paired cell lines. As indicated in Figure 1c, only HDAC1 showed an increasing expression in A549/T cells in comparison with parental cells, suggesting that HDAC1 is a dominant factor for HDAC activity in the acquired paclitaxel-resistant process. In consistence with the increasing HDAC activity, its substrates, including p21, acetylated Histone 3 (Ac-H3), and acetylated Histone 4 (Ac-H4), were also changed in A549/T cells (see Figure 1c), suggesting that HDAC activity is increased in A549/T cells.

To further explore the malignant phenotype characteristics of paclitaxel-resistant cells, A549/T and its parental cells were subcutaneously injected into the SCID mice. Our data showed that $A 549 / T$ cells owned an enhanced tendency to tumorigenicity as compared with A549 cells ( $P=0.04$, Figure $1 \mathrm{~d})$. Simultaneously, A549/T tumors exhibited an increasing HDAC1 expression and a decreasing Ac-H3 and Ac-H4 level (Figure 1e), which indicated that paclitaxel-resistant cells were critically dependent on HDAC activity. In addition, we found that knockdown of HDAC1 by specific siRNA could inhibit the cell viability and sensitize A549/T cells to paclitaxel (Figures $1 f$ and $\mathrm{g}$ ). The above data demonstrate that the increasing HDAC activity results in an enhanced tumorigenicity, a greater proliferation ability, and an increased resistance to paclitaxel in NSCLC cells, giving the rationale for an efficacious therapeutic strategy.
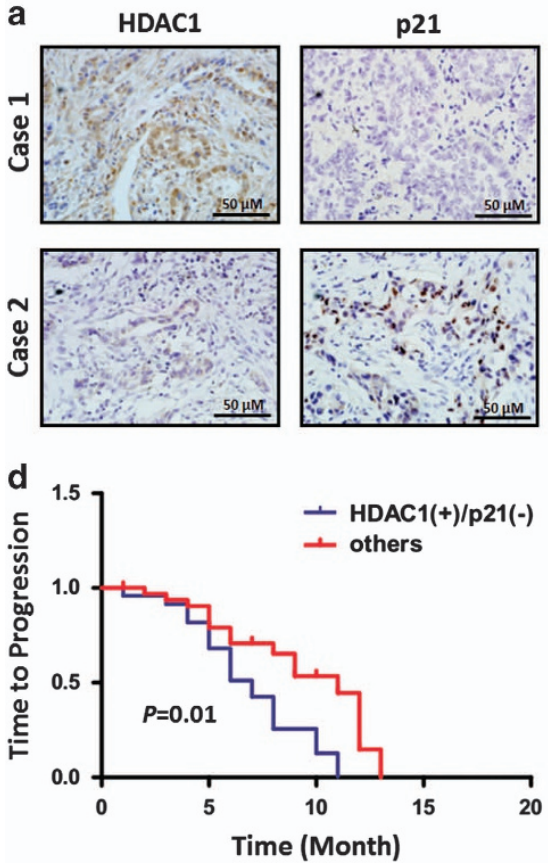

NSCLC tissues with increased HDAC1 and decreased p21 expressions predicted to poor prognosis of patients treated with paclitaxel. We next investigated whether the increasing HDAC activity was detectable in human paclitaxelresistant NSCLC tissue samples. Using immunohistochemistry method, we detected HDAC1 and p21, which was considered as a HDAC target ${ }^{22}$ and was verified to be altered in our in vitro data, in paraffin-embedded tissues from 59 paclitaxel-treated NSCLC patients. Clinico-pathological data indicated that there were no relationship between the expressions of HDAC1 or p21 and major clinicopathological factors (Supplementary Table 1). Additionally, our data showed that there were 32 tumors (54.2\%) showing a higher level of HDAC. Interestingly, among 32 tumors with higher HDAC1, 25 tumors (78.1\%) expressed p21 at a lower level (Figure 2a). Statistical analysis data indicated that there was a negative correlation between HDAC1 and p21 in lung cancer tissues $(P<0.05$, Figure $2 b)$. Importantly, the opposite expression pattern (higher HDAC1 and lower p21) correlated with bad treatment response, short time to tumor progression (TTP) and poor overall survival than other groups in lung cancer (Figures 2c-e). Therefore, HDAC1 along with p21 may serve as a predictive marker for paclitaxel efficacy and their changes may be associated with paclitaxel resistance.

SNOH-3, a novel HDAC inhibitor, induced acetylation of histones and prompted upregulation of p21 in NSCLC cells. We had synthesized a series of HDAC inhibitors (unpublished data). Among them, $\mathrm{SNOH}-3$ exhibited clear inhibitory effects on HDAC activity in vitro with similar capacity to SAHA (Figures $3 a$ and $b$, preparation of
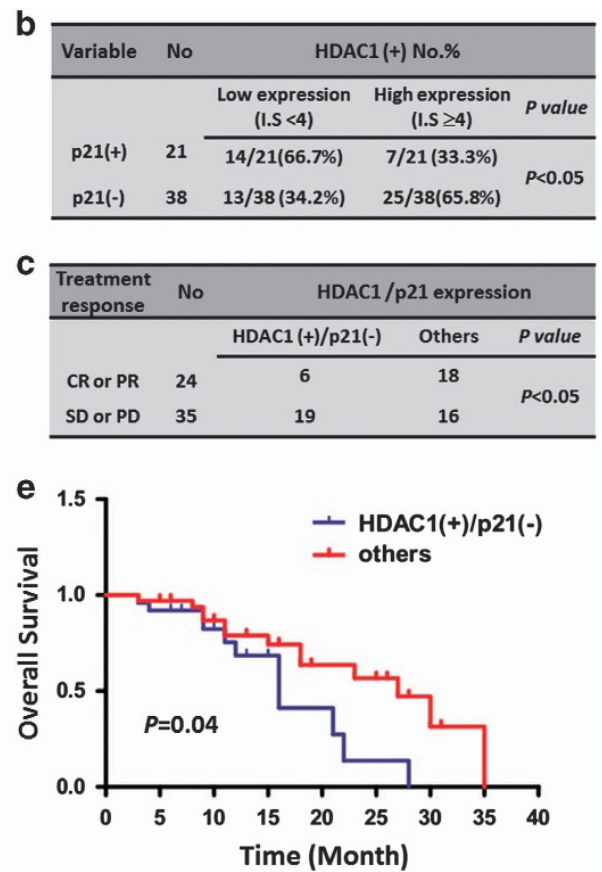

Figure 2 HDAC1 and p21 expression in NSCLCs treated with paclitaxel. (a) The expression of HDAC1 and p21 in representative NSCLC tissues. Figures magnified $\times 400$. (b) The correlation between HDAC1 and p21 in NSCLC. A sample is defined as HDAC1+ if it has an IS of $\geq 4$; p21+ if it has an IS of $\geq 2$. (c) The correlation between treatment response and the expression of HDAC1 along with p21 in NSCLC. CR, complete response; PR, partial response; SD, stable disease; PD, progressive disease. (d) TTP according to expression of HDAC1 along with p21 in NSCLC. (e) Overall survival according to expression of HDAC1 along with p21 in NSCLC 
a
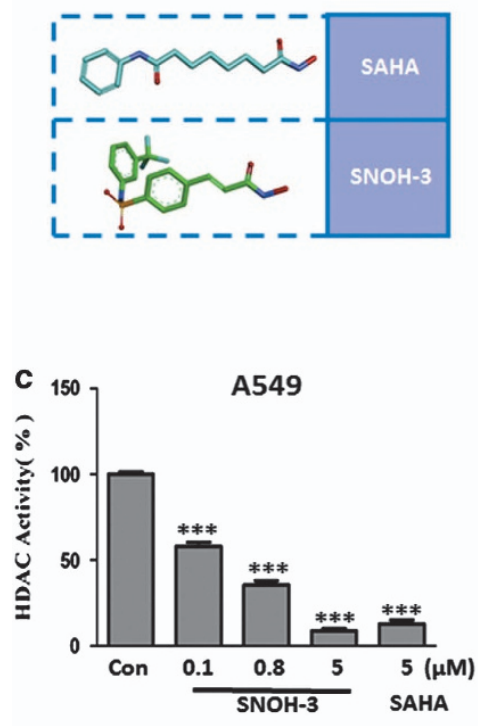

d

\begin{tabular}{|c|c|c|c|c|}
\hline \multirow[b]{2}{*}{ Compounds } & \multicolumn{4}{|c|}{$\mathrm{IC}_{50}(\mathrm{nM})$} \\
\hline & HDAC1 & HDAC3 & HDAC6 & HDAC8 \\
\hline SNOH-3 & $46.5 \pm 13.1$ & $28.1 \pm 2.3$ & $42.3 \pm 11.8$ & $146.2 \pm 52.6$ \\
\hline SAHA & $93.4 \pm 16.9$ & $51.2 \pm 9.7$ & $26.6 \pm 6.3$ & $195.9 \pm 32.5$ \\
\hline
\end{tabular}

e
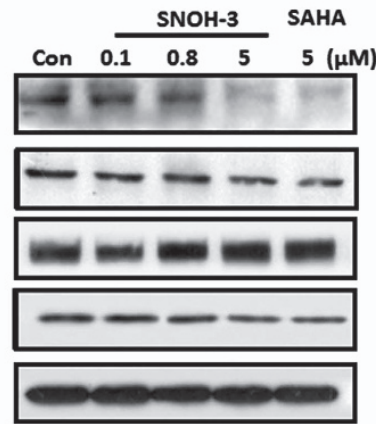

A549

f
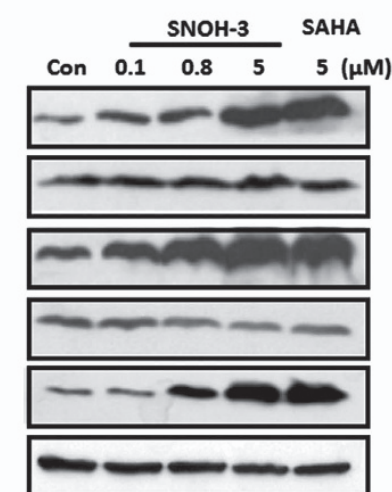

A549
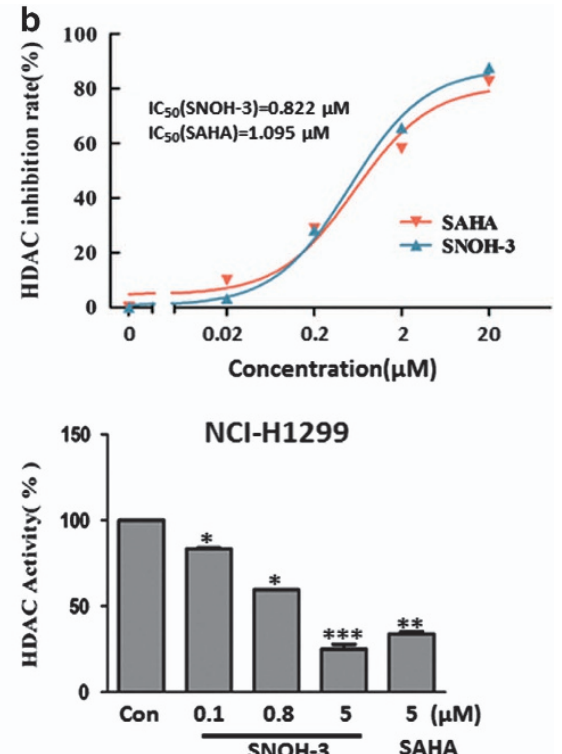

\begin{tabular}{rlllll} 
Con & 0.1 & 0.8 & 5 & 5 & $(\mu \mathrm{M})$ \\
\hline & & &
\end{tabular}

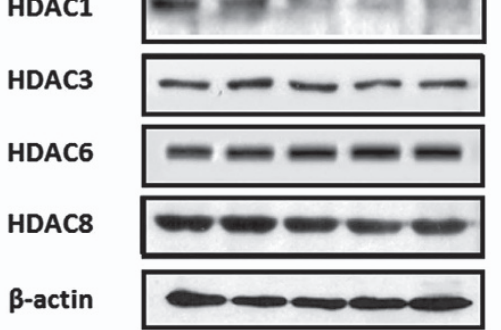

NCl-H1299

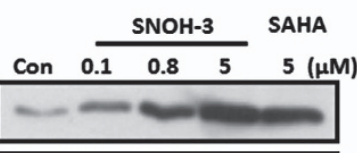

H3

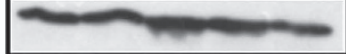

Ac-H4

H4

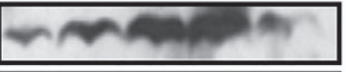

p21

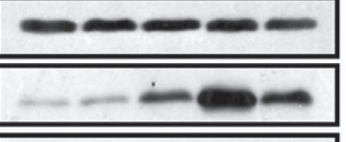

$\beta$-actin

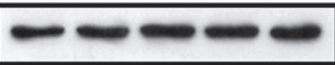

NCl-H1299

Figure 3 The effects of SNOH-3 on HDAC inhibition. (a) 3D structure of SAHA and SNOH-3. (b) In vitro inhibition of HDAC by SNOH-3 and SAHA at different concentrations $(0.02,0.2,2$, and $20 \mu \mathrm{M})$ in cell-free assay. (c) The inhibition of HDAC by various concentrations of SNOH-3 $(0.1,0.8$, and $5 \mu \mathrm{M})$ and SAHA $(5 \mu \mathrm{M})$ in cell-based assay. (d) In vitro inhibition of HDAC isoforms by SNOH-3 and SAHA at different concentrations $(0.01,0.1,1$, and $10 \mu \mathrm{M})$ in cell-free assay. (e) The effects of SNOH-3 on the expression of HDAC1, HDAC3, HDAC6, and HDAC8 in A549 and NCl-H1299 cells after SNOH-3 $(0.1,0.8$, and $5 \mu \mathrm{M})$ treated for $24 \mathrm{~h}$. (f) The effects of SNOH-3 on the expression of Ac-H3, H3, Ac-H4, $\mathrm{H} 4$, and $\mathrm{p} 21$ in $\mathrm{A} 549$ and $\mathrm{NCl}-\mathrm{H} 1299$ cells after SNOH-3 $(0.1,0.8$, and $5 \mu \mathrm{M})$ treated for $24 \mathrm{~h}$. $\beta$-Actin expression was used as a loading control. All error bars are S.E.M. ${ }^{*} P<0.05,{ }^{* *} P<0.01,{ }^{* \star *} P<0.001$ compare with control 
SNOH-3 was provided in Supplementary Materials and Methods). To confirm its inhibition in cells, A549 and a known drug-resistant cells $\mathrm{NCl}-\mathrm{H} 1299$ treated with $\mathrm{SNOH}-3$ or SAHA were analyzed by cell-based HDAC activity. The data showed that SNOH-3 could suppress activity of HDAC in a concentration-dependent manner in both cell lines and displayed a similar potential to SAHA (Figure 3c).

To further explore the characteristics of SNOH-3 on HDAC inhibition, the inhibitory effects of $\mathrm{SNOH}-3$ on HDAC isoforms activity were determined. As shown in Figure 3d, for HDAC-1, -3 , and $-8, \mathrm{SNOH}-3$ exhibited an enhanced inhibitory potential when compared with that of SAHA. However, for HDAC-6, $\mathrm{SNOH}-3$ displayed a lower inhibitory ability in comparison with that of SAHA. Moreover, we also detected the expression level of $\mathrm{HDAC}$ isoforms in $\mathrm{A} 549$ and $\mathrm{NCl}-\mathrm{H} 1299$ cells after treated with both compounds. As shown in Figure $3 e, \mathrm{SNOH}-3$ treatment could result in a concentration-dependent decrease of HDAC1 in both cell lines, suggesting that $\mathrm{SNOH}-3$ could inhibit HDAC1 activity through downregulating its expression. In contrast to HDAC1, other isoforms, including HDAC3, -6, and -8 , were not obviously changed by treatment with $\mathrm{SNOH}-3$ in both cell lines, indicating that the activity inhibition of HDAC-3, -6 , and -8 by SNOH-3 may be not related to their expression regulation.

We subsequently confirmed the HDAC inhibition activity of SNOH-3 by detecting histones acetylation and p21 induction in both cell lines. Parallel to its HDAC inhibition, SNOH-3 significantly increased the levels of acetylated $\mathrm{H} 3$, acetylated H4 and p21 (Figure 3f) in a concentration-dependent manner in both cell lines. At the same concentration, SNOH-3 exhibited a greater capability to induce the acetylation than SAHA, especially in $\mathrm{NCl}-\mathrm{H} 1299$ cells. The above data demonstrate that $\mathrm{SNOH}-3$ is identified as a novel HDAC inhibitor with greater capability than SAHA.

SNOH-3 induced cancer cell apoptosis and inhibited migration, invasion and tube formation of endothelial cells. To assess the antitumor effect of the novel HDAC inhibitor $\mathrm{SNOH}-3$, we next examined the effects of $\mathrm{SNOH}-3$ on cell apoptosis by measuring the cleavages of Caspase-3 and PARP, which are regarded as biomarkers of cell apoptosis. ${ }^{23}$ Our results indicated that $\mathrm{SNOH}-3$ treatment at higher concentration $(5 \mu \mathrm{M})$ resulted in an obvious increase in the cleavages of Caspase- 3 and PARP as compared with control, but there were no significant effects of $\mathrm{SNOH}-3$ at lower concentrations ( 0.1 and $0.8 \mu \mathrm{M}$, Figure $4 \mathrm{a})$. In addition, $\mathrm{SNOH}-3$ exposure also resulted in diminished expression of anti-apoptotic proteins XIAP and Survivin, which might explain the underlying mechanisms of $\mathrm{SNOH}$-3-induced apoptosis. The above data were further confirmed using Annexin V/PI double staining methods. The data revealed that SNOH-3 $(5 \mu \mathrm{M})$ caused an increase in the percentage of apoptosis cells (Annexin V+) in A549 cells (Figure 4b). The role of HDAC inhibition in $\mathrm{SNOH}-3$-induced apoptosis was confirmed by silencing HDAC1 in A549 cells. The SNOH-3caused increase in apoptosis was partially blocked by specific HDAC1 siRNA (Figure 4b).

The migration, invasion, and angiogenesis of endothelial cell are inevitable to solid tumor growth and metastasis. ${ }^{24}$ Therefore, we also evaluated the effects of $\mathrm{SNOH}-3$ on
VEGF-induced migration, invasion, and tube formation of human umbilical vein cells (HUVECs) in vitro. To exclude the cytotoxicity of $\mathrm{SNOH}-3$, we first examined the effect of SNOH-3 on the viability of HUVECs. SNOH-3 treatment for $24 \mathrm{~h}$ caused a slight decrease in the percentage of viable HUVECs at $5 \mu \mathrm{M}$ with inhibition rate lower than $15 \%$ (Figure 4c). Thus, the maximal concentration of $5 \mu \mathrm{M}$ was determined in the following experiments. The effect of SNOH-3 on the migration of HUVECs was detected by real-time cell analysis (RTCA) assay. Our data indicated that SNOH-3 obviously reduced VEGF-induced HUVECs migration (Figure 4d), whereas SAHA did not display an inhibitory effect. In addition, transwell invasion assay showed that SNOH-3 dramatically suppressed VEGF-induced HUVECs invasion (Figure 4e). Next, we investigated how SNOH-3 affected HUVEC tube formation. As shown in Figure 4f, in the presence of VEGF, elongated and robust tube-like structures were formed. When exposed to $\mathrm{SNOH}-3$, the formation of tubular structures, especially branch points, was significantly inhibited. Both inhibitory effects of $\mathrm{SNOH}-3$ were concentration dependent, and $\mathrm{SNOH}-3$ exhibited a relatively enhanced ability to inhibit invasion and tube formation of HUVECs as compared with SAHA.

To explore the underlying mechanism of SNOH-3-caused inhibition of migration, invasion, and tube formation in HUVECs, we measured the expression level of VEGF in HUVECs after exposure to SNOH-3. The results revealed that the protein expression of VEGF was downregulated by SNOH-3 treatment in HUVECs (Supplementary Figure 1).

Previous study reported that KLF4, a transcription repressor of $\mathrm{VEGF},{ }^{25}$ was regulated by HDAC activity, ${ }^{26}$ thus we also assessed the effect of SNOH-3 on the expression of KLF4 in HUVECs. Our data indicated that SNOH-3 could increase the expression of KLF4 in a concentration-dependent manner in HUVECs (Supplementary Figure 1). These results indicated that $\mathrm{SNOH}-3$ might suppress cell migration, invasion, and angiogenesis through the induction of KLF4 expression to reduce VEGF.

SNOH-3 combined with paclitaxel overcome paclitaxel resistance in vitro via inducing apoptosis. Because HDAC1 was overexpressed in paclitaxel-resistant A549 (A549/T) cells and its silence could sensitize A549/T cells to paclitaxel, the growth inhibitory effect of $\mathrm{SNOH}-3$ in combination with paclitaxel on A549/T cells was assessed. As shown in Figure $5 \mathrm{a}$, the combination of $\mathrm{SNOH}-3$ and paclitaxel could obviously inhibit cell proliferation as compared with SNOH-3 or paclitaxel alone. The synergistic action analysis data indicated that the combination led to a strong synergistic antiproliferative activity on A549/T cells (Figure $5 \mathrm{~b}$ ), and the $\mathrm{ED}_{50} \mathrm{Cl}$ was 0.366 . In addition, we also found that $\mathrm{SNOH}-3$ treatment could suppress the HDAC activity in a concentration-dependent manner in A549/T cells (Figure $5 \mathrm{c}$ ), suggesting that HDAC inhibition might have a crucial role in this synergistic effect.

Corresponding to the synergistic inhibition on cell proliferation, $\mathrm{SNOH}-3$ plus paclitaxel could result in an increased cleavages of PARP and caspase- 3 as compared with paclitaxel or $\mathrm{SNOH}-3$ alone (Figure $5 \mathrm{~d}$ ), suggested that the 
a

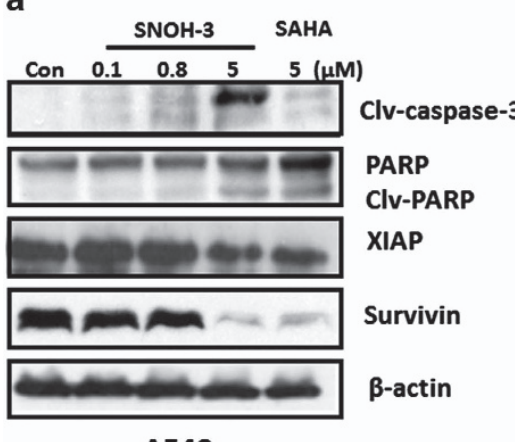

A549

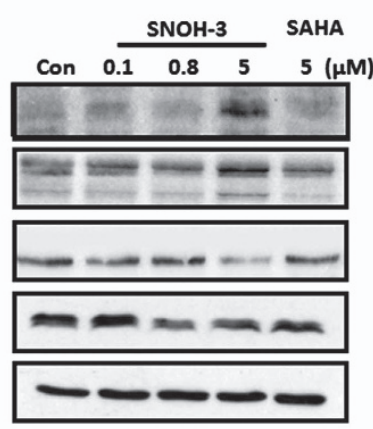

NCl-H1299 b

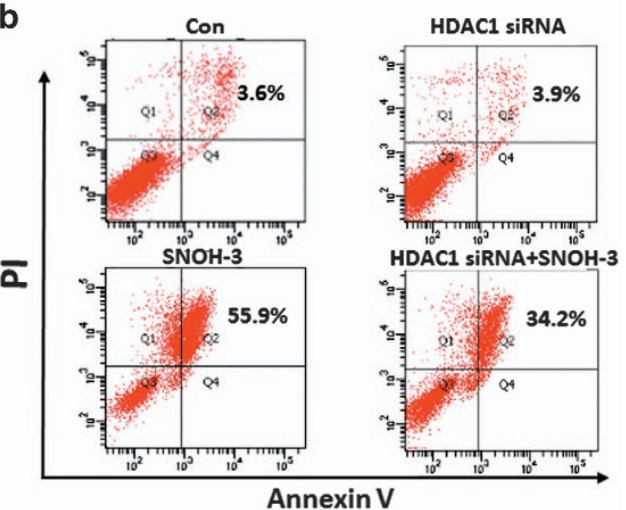

C

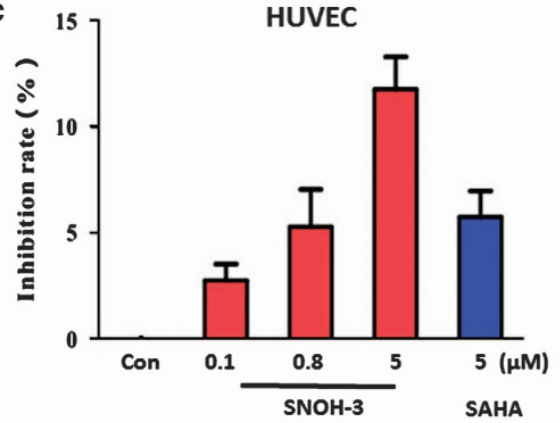

d

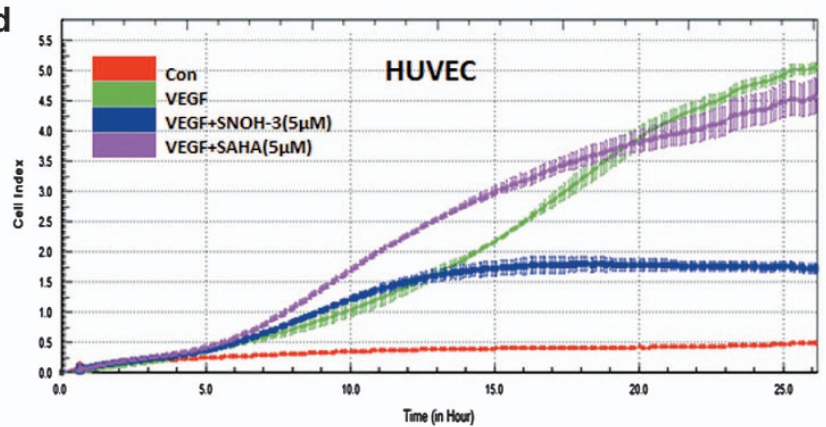

e
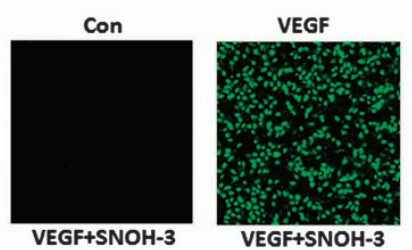

$(0.1 \mu \mathrm{M})$

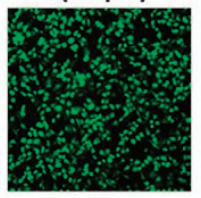

$(0.8 \mu \mathrm{M})$

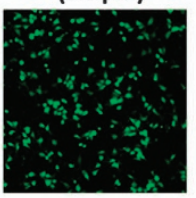

VEGF+SAHA (5 $\mu M)$

VEGF+SNOH-3

$(5 \mu \mathrm{M})$
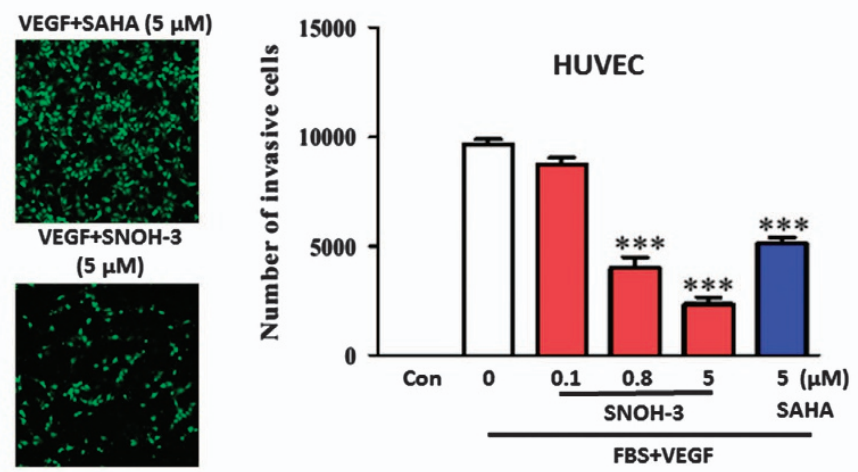

f

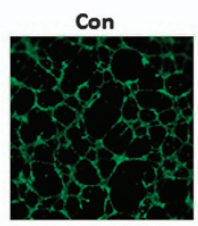

VEGF+SNOH-3

(0.1 $\mu \mathrm{M})$

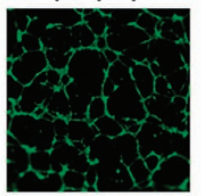

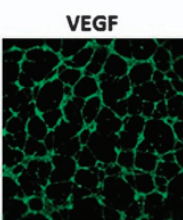

VEGF+SNOH-3 $(0.8 \mu \mathrm{M})$

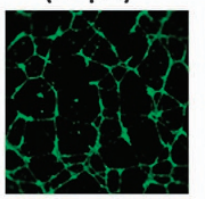

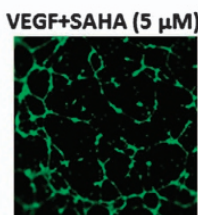

VEGF+SNOH-3 (5 $\mu \mathrm{M})$

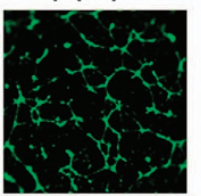

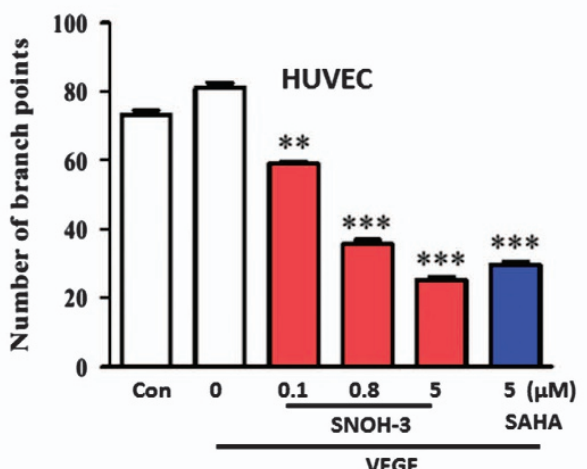

Figure 4 The effects of SNOH-3 on apoptosis of tumor cells and VEGF-induced migration, invasion, and tubular structure formation of endothelial cells. (a) The apoptosisrelated proteins (clv-caspase-3, PARP, XIAP, and Survivin) were assessed by western blot analyses in A549 and NCl-H1299 cells after SNOH-3 (0.1,0.8, and $5 \mu \mathrm{M})$ treated for $48 \mathrm{~h}$. $\beta$-Actin expression was used as a loading control. (b) The apoptotic cells were assessed by FACS analyses in A549 cells after SNOH-3 (5 $\mu$ M) or/and HDAC1 siRNA $(50 \mathrm{nM})$ treated for $48 \mathrm{~h}$. (c) The effect of SNOH-3 on cell viability of HUVECs. The HUVECs were treated with SNOH-3 $(0.1,0.8$, and $5 \mu \mathrm{M})$ or SAHA $(5 \mu \mathrm{M})$ for $24 \mathrm{~h}$. (d) HUVECs migration was measured by RTCA assay after SNOH-3 $(5 \mu \mathrm{M})$ or SAHA $(5 \mu \mathrm{M})$ treated for $24 \mathrm{~h}$. (e) HUVECs invasion was measured by transwell assay after SNOH-3 $(0.1,0.8$, and $5 \mu \mathrm{M})$ or SAHA $(5 \mu \mathrm{M})$ treated for $24 \mathrm{~h}$. (f) HUVECs tube formation was measured after SNOH-3 $(0.1,0.8$, and $5 \mu \mathrm{M})$ or SAHA $(5 \mu \mathrm{M})$ treated for $24 \mathrm{~h}$. All error bars are S.E.M. ${ }^{* *} P<0.01,{ }^{* \star} P<0.001$ compare with control or VEGF control 
a
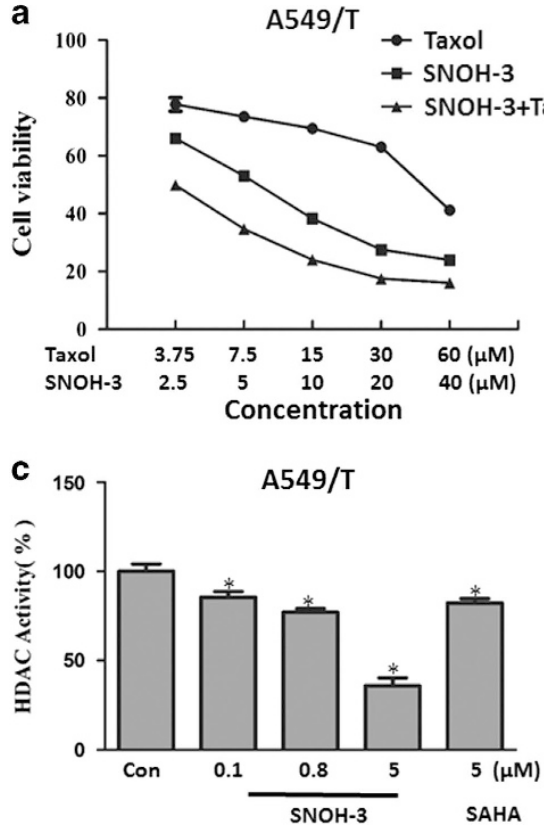

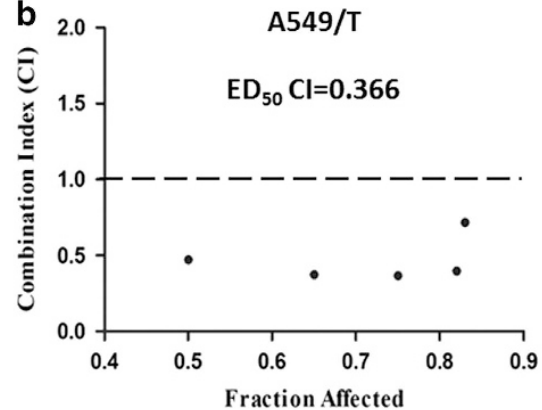

d

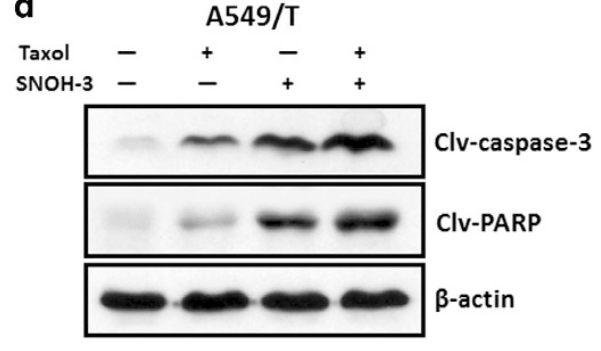

Figure 5 The effects of SNOH-3, paclitaxel, and their combination on the growth and apoptosis of A549/T cells. (a) The growth curve of A549/T cells after treated with SNOH-3, paclitaxel, and the combination of SNOH-3 and paclitaxel. (b) Analysis of the combination of SNOH-3 and paclitaxel in A549/T cells. The cells were treated for $48 \mathrm{~h}$ using increasing concentrations of $\mathrm{SNOH}-3$ and paclitaxel, either alone or in a fixed ratio. The resultant data were analyzed using Calcusyn program, and graphs from the averaged results of three independent experiments are shown. (c) The HDAC activity of A549/T cells was measured by cell-based HDAC assay after SNOH-3 (0.1, 0.8 , and $5 \mu \mathrm{M})$ or SAHA $(5 \mu \mathrm{M})$ treated for $24 \mathrm{~h}$. (d) The apoptosis was assessed by western blotting after SNOH-3 $(10 \mu \mathrm{M})$ or/and SAHA $(15 \mu \mathrm{M})$ treated for $48 \mathrm{~h}$. Cleavages of caspase-3 and PARP were considered as apoptosis. ${ }^{*} P<0.05$ compare with control

synergistic antiproliferation effect of $\mathrm{SNOH}-3$ and paclitaxel on A549/T cells might be due to induction of apoptosis.

SNOH-3 combined with paclitaxel abrogated paclitaxel resistance in vivo through inducing apoptosis and suppressing angiogenesis. We next evaluated the effects of this combination strategy in vivo. NOD/SCID mice bearing A549/T xenografts were treated with paclitaxel alone, $\mathrm{SNOH}-3$ alone, or dual combinations (SNOH-3 plus paclitaxel). Xenografts treated with paclitaxel showed a weak inhibition, which verifying a resistant characteristic (Figure 6a). On the contrary, $\mathrm{SNOH}-3$ administration could result in an obvious suppression of tumor growth during the course of the experiment (2 weeks, Figure 6a). Remarkably, mice treated with the dual combination exhibited a robust inhibition of tumor growth, compared with mice treated with SNOH-3 and paclitaxel alone, therefore mirroring our in vitro results (Figure 6a). In addition, all mice tolerated the treatment well without significant toxicity and showed stable body weights (Figure 6b). Inhibitory effect of $\mathrm{SNOH}-3$ and dual combination on HDAC was confirmed by testing the expression levels of HDAC1, Ac-H3, and p21 in tumor cells obtained from xenografts (Figure 6c). Moreover, in agreement with our in vitro results, xenografts treated with the dual combination displayed an increased apoptosis (cleaved caspase-3 and terminal deoxynucleotide transferasemediated dUTP nick end labeling (TUNEL), Figures $6 \mathrm{c}$ and d) and a decreased angiogenesis (MVD, Figure 6e) in comparison with paclitaxel or SNOH-3-treated animals (Figures $6 \mathrm{c}$ and $\mathrm{d}$ ). These results indicated that $\mathrm{SNOH}-3$ combined with paclitaxel could overcome paclitaxel resistance in vivo via the inhibition of HDAC activity to induce apoptosis and suppress angiogenesis.

\section{Discussion}

Based on demonstrated favorable risk-benefit profiles, paclitaxel remains a key component in the first-line standard of care for advanced NSCLC. ${ }^{5}$ However, the emergence of acquired resistance is inevitable and remains the major obstacle. Delineation of these resistance mechanisms will pave the way for rationally designed and hopefully long-lasting combinations of targeted therapies. In the present study, we found that HDAC activation inhibits its targets expression, such as p21, in paclitaxel-resistant NSCLC cells that lead to the enhancement of proliferation and tumorigenesis, thus dampening the response to the paclitaxel. Furthermore, in clinical NSCLC specimens, HDAC activity was increased in paclitaxel-treated tumors with drug resistance and poor survival. Importantly, our results indicate that HDAC1 inhibition could be a strategy to overcome paclitaxel-acquired resistance. Overall, our results have important implications for clinical oncology and lung cancer biology.

Until now, there were 18 mammalian HDAC isoforms have been identified in humans, which could be divided into four classes according to their homology to yeast prototypes. ${ }^{14}$ Among all of the HDACs, HDAC1, $-3,-6$, and -8 have been frequently revealed to be closely related to tumorigenesis. ${ }^{14}$ Here, we found that HDAC activity was increased in paclitaxelresistant NSCLC cells in comparison with parental cells, and 

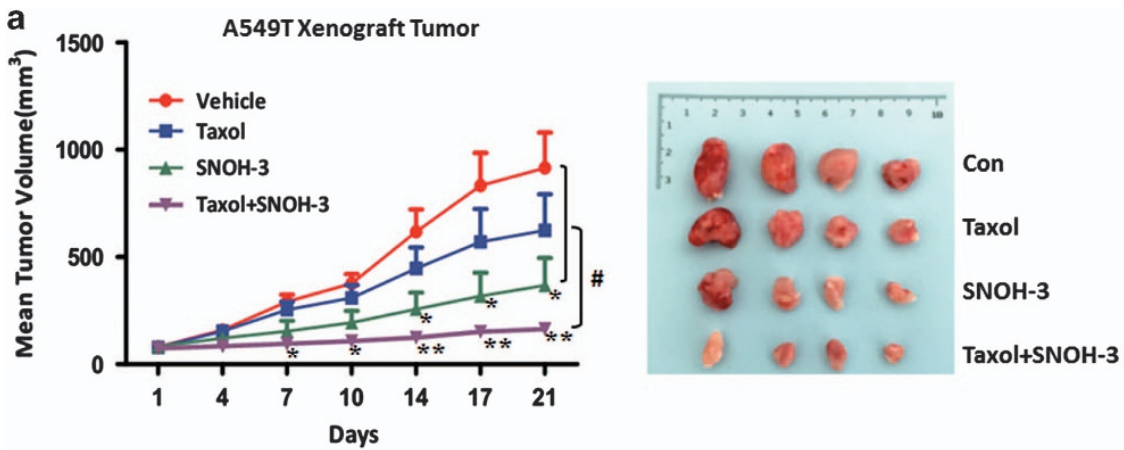

b

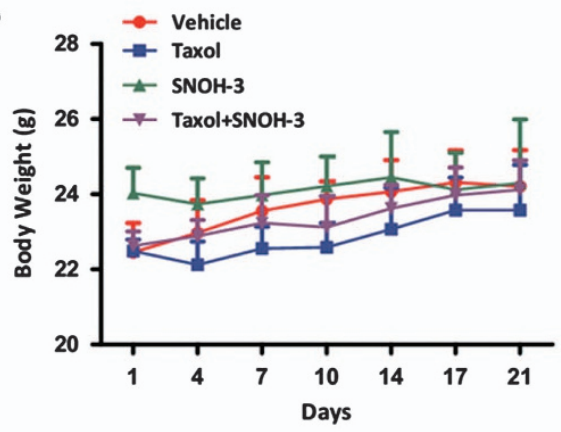

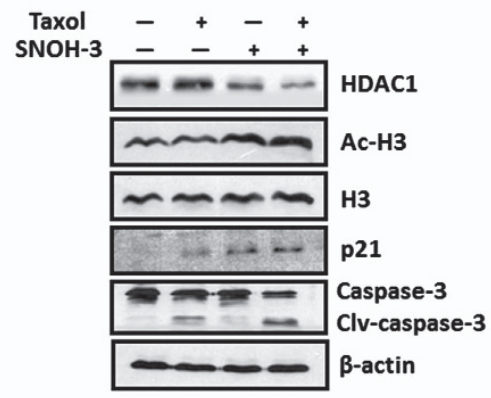

d
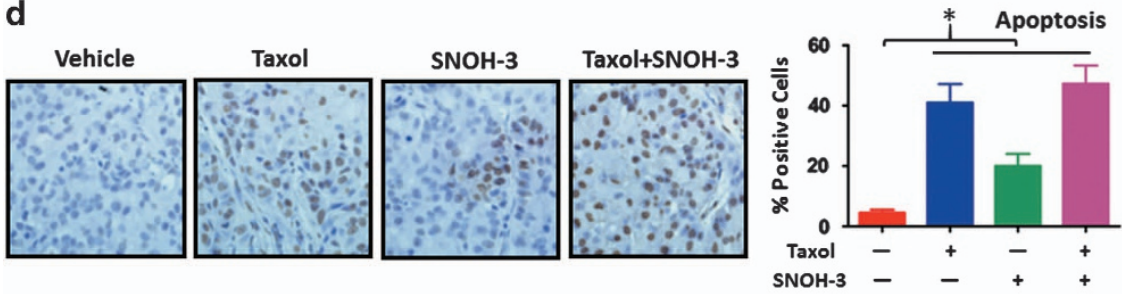

e
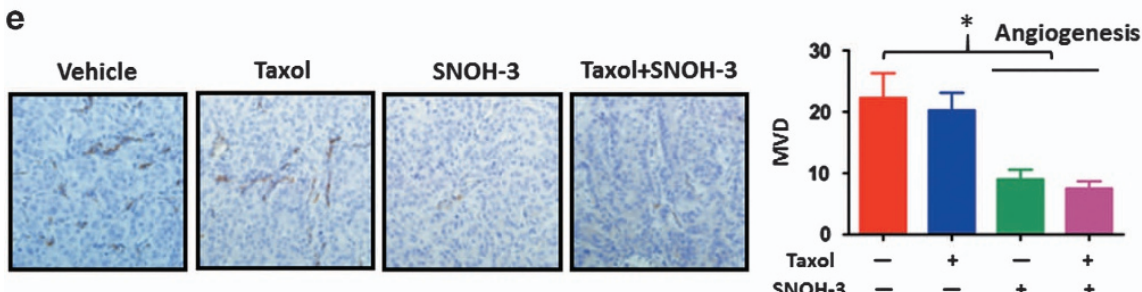

Figure 6 The antitumor effect of SNOH-3 and paclitaxel on A549/T human xenograft models. (a) The mice transplanted with A549/T human xenografts were randomly divided into four groups and given injection of SNOH-3 (40 mg/kg/3 days), paclitaxel (10 mg/kg/week), and the combination or vehicle for a period of 3 weeks. The tumor volumes are expressed as mean \pm S.D. ( $n=4$ per group). (b) The average body weight of each group is expressed as mean \pm S.D. ( $n=4$ per group). (c) Expression of HDAC1, Ac-H3, H3, p21, caspase-3, and PARP extracted from the tumor tissues of drug-administrated mice of four groups was detected by western blotting. (d) The effects of SNOH-3, paclitaxel, and the combination on apoptosis of A549/T xenograft tumors were measured by TUNEL. (e) The effects of SNOH-3, paclitaxel, and the combination on MVD of A549/T xenograft tumors were measured by immunohistochemistry. All error bars are S.E.M. ${ }^{*} P<0.05,{ }^{\star \star} P<0.01, \mathrm{SNOH}-3$, paclitaxel single administration or the combination administration compared with the control, ${ }^{\#} P<0.05$, the combination administration compared with $\mathrm{SNOH}-3$, paclitaxel single administration or vehicle

that resulted in an enhanced proliferation and tumorigenesis capability, suggesting a novel biology role of HDAC in drug resistance. Furthermore, the prognosis significance of HDAC in paclitaxel administrated NSCLCs implies that HDAC might serve as a predictive biomarker for paclitaxel treatment. Interestingly, our data showed that only HDAC1, but not HDAC3 and HDAC8, was overexpressed in A549/T cells, which displayed an increased HDAC activity. This could be explained by the following fact: first, several studies have demonstrated that HDAC1 is a dominant isoform in determining HDAC activity. ${ }^{27,28}$ Second, there are several literatures showed that HDAC1 was overexpressed in multidrug resistance cells. ${ }^{19,29,30}$ Third, loss of HDAC1 usually results in a compensatory increase in the related HDACs. ${ }^{27}$ So it is possible that HDAC1 overexpression would result in the loss of other HDAC isoforms. However, it will be important to fully understand what dictates drug resistance by this understudied HDAC1. Much remains to be determined regarding the interregulation of HDAC isoforms in the process of paclitaxel resistance.

The crucial role of HDAC in NSCLC paclitaxel resistance in our in vitro, in vivo, and clinical tissues studies and limitation of 
FDA-approved HDAC inhibitors in solid cancer prompted us to develop novel HDAC inhibitors. Here, we developed a novel HDAC inhibitor, SNOH-3, which showed more antitumor potent than SAHA. For total activity of HDACs, SNOH-3 showed a similar potent to SAHA, whereas for HDAC1 and HDAC3, SNOH-3 exhibited a greater capability than SAHA. The enhanced capability also was presented in the activation of its substrates. In comparison with SAHA, SNOH-3 displayed a greater ability to increase the expression of $\mathrm{Ac}-\mathrm{H} 3, \mathrm{Ac}-\mathrm{H} 4$, and p21 in NSCLC cells, especially in $\mathrm{NCl}-\mathrm{H} 1299$ cells, which is considered as a drug-resistant cell line. In consistent with HDAC activity data, the functional studies also indicated that $\mathrm{SNOH}-3$ induced a greater apoptosis in NSCLC cells when compared with those of SAHA, and HDAC1 had an important role in this process. Mechanistically, we found that $\mathrm{SNOH}-3$ reduces the antiapoptosis protein Survivin and XIAP, which could be explained by the fact that Survivin and XIAP are also the targets of HDAC inhibitor, ${ }^{31,32}$ so it is possible that $\mathrm{SNOH}-3$ induces apoptosis through reducing the expression of Survivin and XIAP. Furthermore, our results showed that $\mathrm{SNOH}-3$ exhibited an obvious inhibition to VEGF-induced migration, invasion, and angiogenesis in HUVECs. The underlying mechanisms might be that $\mathrm{SNOH}-3$ treatment resulted in HDAC inhibition, then led to upregulation of KLF4 which is considered as a transcription repressor of VEGF, ${ }^{25,26}$ finally contributed to the downregulation of VEGF.

Among the mechanisms of paclitaxel resistance, alteration of drug target (tubulin), overexpression of drug efflux pumps, and activation of HIF-1 signaling are widely recognized. Here, we found that HDAC was also involved in the paclitaxel resistance. The inner link between HDAC and the classical resistant mechanisms might be illustrated by the previous literatures: (1) Dowdy et al. $^{33}$ reported that TSA, an HDAC inhibitor, stabilized microtubules via $a$-tubulin acetylation in endometrial cancer cells and enhanced paclitaxel effects. (2) Kaewpiboon et al. ${ }^{34}$ demonstrated that P-glycoprotein (Pgp), a well-known drug efflux pump, could be regulated by HDAC at the transcriptional level in lung cancer cells. (3) Several groups showed that HDAC inhibitors could inhibit HIF-1 activity by modulating its interactions and its acetylation status. $^{35,36}$ The above-reported results indicated that HDAC might be also involved in other paclitaxel-resistant mechanisms. However, the detail role of HDAC in these process need be further illustrated.

Importantly, although resistance to paclitaxel in NSCLC has been a subject of considerable interest, the development of therapeutic strategies that overcome paclitaxel resistance has remained an elusive challenge in clinical oncology. The increase of HDAC activity in our in vitro and in vivo models prompted us to evaluate the combination strategy that abrogated paclitaxel resistance in vitro and in vivo. Our data indicated that $\mathrm{SNOH}-3$ in combination with paclitaxel exerted promising activity to overcome paclitaxel resistance in vitro and in preclinical models through induction apoptosis and inhibition angiogenesis. These data corroborate previous study in clinical, which have found an enhanced efficacy of HDAC inhibitor combined with carboplatin and paclitaxel in patients with advanced NSCLC. ${ }^{21}$ In summary, given the limited capacity of paclitaxel to control advanced NSCLC, this work lays the foundation for a promising therapeutic strategy.

In conclusion, a common and devastating phenomenon in clinical oncology is acquired drug resistance. We provide evidence that the increase in HDAC activity was found from NSCLC with paclitaxel-acquired resistant cells, and HDAC1 overexpression along with p21 downregulation predicted bad response, short TTP, and poor overall survival of NSCLC patients with paclitaxel treatment. Knockdown of HDAC1 could sensitize paclitaxel-resistant cells to paclitaxel and inhibit cell proliferation. The combination of HDAC inhibitor SNOH-3 with paclitaxel inactivated HDAC, and thus conferred its proapoptotic and antiangiogenic effects in paclitaxel-resistant models. Our finding not only elucidated an additional mechanism for acquired paclitaxel resistance in NSCLC, but also provided a promising therapeutic strategy to overcome paclitaxelacquired resistance.

\section{Materials and Methods}

Cell lines and cell culture. Human lung cancer cell lines A549 and $\mathrm{NCl}-$ H1299, and HUVEC were obtained from the American Type Culture Collection (Manassas, VA, USA). A549/paclitaxel resistance cells (A549/T) were obtained from Key GEN Bio Tech (Nanjing, China, CHN). These cancer cells were routinely cultured in RPMI- 1640 or MEM supplemented with $10 \%$ fetal bovine serum (FBS) and maintained at $37{ }^{\circ} \mathrm{C}$ in a humidified incubator with $5 \% \mathrm{CO}_{2}$. Specially, the resistance of $A 549 / T$ cells was maintained by continuously exposing to paclitaxel $(200 \mathrm{ng} / \mathrm{ml})$. HUVECs were maintained as a monolayer in MCDB131 medium supplemented with $20 \%$ (v/v) FBS, $1 \%$ (v/v) L-glutamine, 5 units $/ \mathrm{ml}$ heparin, and $50 \mathrm{mg} / \mathrm{ml}$ endothelial cell growth factor (ECGF) using culture flasks or plates precoated with $1 \%(\mathrm{v} / \mathrm{v})$ gelatin.

Patients and chemotherapy. A total of 56 patients with advanced NSCLC (stage IIIB and stage IV) were enrolled between January 2004 and June 2012 from Wuhan General Hospital of Guangzhou Command (Wuhan, PR China). The enrolled patients met the following eligibility criteria: histological or cytological confirmation of NSCLC, the presence of measurable disease, no adjuvant or neoadjuvant therapy and surgery, no second malignancies, availability of adequate diagnostic tumor tissue (taken from brochoscopic biopsy, percutaneous lung biopsy or metastatic sites). The clinicopathologic features of these patients have been summarized in Supplementary Table 1.

Each patient underwent the treatment with at least two cycles of the first-line platinum-paclitaxel chemotherapy. Response to treatment was determined after 2-3 cycles by RECIST (Response Evaluation Criteria in Solid Tumors) criteria, which classified the responses into complete response (CR), partial response (PR), stable disease (SD), and progressive disease (PD). SD plus PD were considered as chemotherapy resistance. TTP was defined as the time between the onset of chemotherapy and the date of first documented disease progression. Overall survival (OS) was defined as the time between the onset of chemotherapy and the date of the last follow-up or death from any cause. Ethical oversight and approval was obtained from the Institutional Review Board of Wuhan General Hospital of Guangzhou Command.

Immunohistochemistry. A tissue microarray (TMA) was constructed (in collaboration with the Shanghai Biochip Company Ltd., Shanghai, China, CHN) as described previously. ${ }^{17}$ Following antigen retrieval and blocking, TMA sections $(4 \mathrm{~mm})$ were immunostained using antibodies against HDAC1 (1:200 dilution; Abcam, Cambridge, UK) and p21 $1^{\text {Wat1/Cip1 }}$ (1:50 dilution; Cell Signaling Technology, Danvers, MA, USA) and with detection using the avidin-biotin complex method (DAKO) visualized by $3,3^{\prime}$-diaminobenzidine (DAB). Slides were lightly counterstained with hematoxylin. The evaluation of both the intensity of immunohistochemical staining and the proportion of positively stained epithelial cells was previously described. ${ }^{33}$

Compounds and reagents. SNOH-3, with purity $>98 \%$, was synthesized in Medicine Chemistry Laboratory at Shenyang Pharmaceutical University (see Figure $3 \mathrm{a}$ and Supplementary data). SAHA were obtained from Sigma 
(St. Louis, MO, USA). These agents were dissolved in DMSO to $100 \mathrm{mM}$ and stored at $-20^{\circ} \mathrm{C}$. Before treatment, the stock solution is diluted to different concentrations. The final concentration of DMSO in cultures is $0.1 \%(\mathrm{v} / \mathrm{v})$ or less. MTT (3-(4,5dimethylthiazol-2-yl)-2,5-diphenyl tetrazolium bromide) and propidium idodide (PI) were purchased from Sigma. The primary antibodies against HDAC1, HDAC3, HDAC6, HDAC8, Histone 3, Histone 4, p21 WAF1/CIP1, PARP, Caspase-3, Survivin, $\mathrm{XIAP}, \mathrm{KLF} 4$, VEGF, and $\beta$-actin were got from Cell Signaling Technology. The primary antibodies against CD31 were obtained from Santa Cruz Biotechnology (Santa Cruz, CA, USA). The primary antibodies against Ac-H3 and Ac-H4 were purchased from Millipore (Boston, MA, USA). The HDAC1 Silencer Select Validated siRNA was got from Life Technologies (Waltham, MA, USA).

Cell viability assay. The in vitro cell viability was determined by MTT assay. The cells $\left(1 \times 10^{5} \mathrm{cells} / \mathrm{ml}\right)$ were seeded into 96 -well culture plates. After overnight incubation, the cells were treated with various concentrations of agents for 24 or $48 \mathrm{~h}$. Then $10 \mu \mathrm{l}$ MTT solution ( $2.5 \mathrm{mg} / \mathrm{ml}$ in PBS) was added to each well, and the plates were incubated for an additional $4 \mathrm{~h}$ at $37^{\circ} \mathrm{C}$. After centrifugation (2500 r.p. $\mathrm{m} ., 10 \mathrm{~min}$ ), the medium with MTT was aspirated, followed by the addition of $100 \mu \mathrm{l}$ DMSO. The optical density of each well was measured at $570 \mathrm{~nm}$ with a Biotek Synergy HT Reader (Winooski, VT, USA).

HDAC activity assay. The in vitro HDAC assay was performed with an HDAC fluorescent activity assay kit (BioVision, San Francisco, CA, USA) as our previous reported. ${ }^{17}$ Briefly, A549 nuclear proteins were incubated with different concentrations of compounds and SAHA (Sigma) at $37^{\circ} \mathrm{C}$ for $30 \mathrm{~min}$ in the presence of an HDAC fluorimetric substrate. The HDAC assay developer (which produces a fluorophore in reaction mixture) was added, and the fluorescence was measured using a microplate reader (Molecular Devices, Sunnyvale, CA, USA). For cell-based HDAC activity assay, the A549, A549/T, and NCl-H1299 cells were treated with different concentrations of SNOH-3 or SAHA for $24 \mathrm{~h}$ before assays. Proteins were isolated by using cell lysis buffer (Beyontime, Guangdong, China, $\mathrm{CHN}$ ). The protein concentration was measured by BCA protein assay (Beyontime, $\mathrm{CHN}$ ). The other procedure is same as in vitro HDAC assay. HDAC activity is presented as the means \pm S.E.M. of three determinants.

HDAC isoforms activity assay. The HDAC1, 3, 6, and 8 activity was assessed using commercial kits (BPS Bioscience, San Diego, CA, USA). Briefly, the purified HDAC proteins were incubated with different concentrations of SNOH-3 and SAHA at $37^{\circ} \mathrm{C}$ for $30 \mathrm{~min}$ in the presence of an HDAC fluorimetric substrate containing an acetylated lysine side chain. The HDAC assay developer (which produces a fluorophore in reaction mixture) was added, and the fluorescence was measured using a microplate reader (Molecular Devices). HDAC isoforms activity is presented as the means \pm S.E.M. of three determinants.

Western blot analysis. About $1 \times 10^{7}$ cells were gathered after pre-treatment for the indicated time periods as described previously. Western blotting was performed as previously described. ${ }^{17}$ Briefly, an equal amount of total protein extracts from cultured cells or tissues was fractionated by 10-15\% SDS-PAGE and then electrically transferred onto polyvinylidene difluoride (PVDF) membranes. Mouse or rabbit primary antibodies and appropriate horseradish peroxidase (HRP)conjugated secondary antibodies were used to detect the designated proteins. The bound secondary antibodies on the PVDF membrane were reacted with ECL detection reagents (Pierce, Rockford, IL, USA) and exposed to X-ray films. Results were normalized to the internal control $\beta$-actin.

Flow-cytometry analysis. Analyses for apoptosis were also conducted with an Annexin V-FITC Apoptosis Detection Kit (BioVision). A549 cells $\left(1 \times 10^{6}\right)$ were exposed to SNOH-3 $(5 \mu \mathrm{M})$ or/and HDAC1 siRNA $(50 \mathrm{nM})$ for $48 \mathrm{~h}$. They were collected by centrifugation and resuspended in $500 \mu \mathrm{l}$ of $1 \times$ binding buffer. Annexin V-fluorescein isothiocyanate (FITC; $5 \mu \mathrm{l})$ and PI $(5 \mu \mathrm{l})$ were added to the cells. After incubation at room temperature for $5 \mathrm{~min}$ in the dark, cells were analyzed by FACS using a flow cytometer (Becton Dickinson, Bergen, NJ, USA). Cells that stained Annexin V-FITC were analyzed.

Real-time cell analysis. The CIM-plate contains 16 modified Boyden chambers, which can be used independently to measure cell migration in real time through $8 \mu \mathrm{m}$ pores of a polyethylene terephthalate membrane onto gold electrodes on the underside of the membrane using the XCELLigence analyser system (ACEA Biosciences, San Diego, CA, USA). Experiments were set up according to the manufacturer's instructions with the membrane uncoated (migration). A chemotactic signal for movement was provided by inoculating 30 000-50 000 HUVECs in serum-free medium in the upper chamber and supplying $10 \% \mathrm{FBS}$ in the lower chamber $(10 \mathrm{ng} / \mathrm{ml}$ VEGF with the relevant concentration of drug). Cell index (electrical impedance) was monitored every $5 \mathrm{~min}$ for the duration of the experiment. The cell index represents the capacity for cell migration, and the slope of the curve can be related to the migration velocity of tumor cells. The cell index thus reflects the tumor cell's migratory capacity.

Transwell invasion assay. Invasion of HUVECs was assayed using Transwell (Corning Costar, Corning, NY, USA) with $6.5-\mathrm{mm}$ diameter polycarbonate filters (8- $\mu \mathrm{m}$ pore size). Briefly, the lower surface of the filter was coated with Matrigel. Fresh M200 medium (1\% FBS) containing $10 \mathrm{ng} / \mathrm{ml}$ VEGF was placed in the lower wells. The cells were trypsinized and suspended at a final concentration of $1 \times 10^{6} \mathrm{cells} / \mathrm{ml}$ in M2O0 containing $1 \%$ FBS. Various concentrations of SNOH-3 and SAHA were given to the cells for $30 \mathrm{~min}$ at room temperature before seeding. One hundred microliters of the cell suspension were loaded into each of the upper wells, and the chamber was incubated at $37^{\circ} \mathrm{C}$ for $12 \mathrm{~h}$. The cells were fixed and stained with Calcein-AM. Non-invasion cells on the upper surface of the filter were removed by wiping with a cotton swab, and chemotaxis was quantified with a high content drug screening system ImageXpress ${ }^{\mathrm{R}}$ Micro (Molecular Devices) by counting cells that had migrated to the lower side of the filter.

Tube formation assay. HUVECs was incubated in M200 containing $2 \%$ LSGS (Low Serum Growth Supplement). Various concentrations of SNOH-3 and SAHA were added to the cells for $30 \mathrm{~min}$ at room temperature before seeding. Cells were plated onto the layer of Matrigel at a density of $1.8 \times 10^{5}$ cells per well, followed by the addition of $10 \mathrm{ng} / \mathrm{ml}$ VEGF. After $12 \mathrm{~h}$, the cells were fixed and stained with Calcein-AM. The tube branch points were determined using the high content drug screening system ImageXpress ${ }^{\mathrm{R}}$ Micro (Molecular Devices) after photographed (x400).

Determination of combination index. A549/T cells were treated with different concentrations of single SNOH-3, paclitaxel, or their combination. The cell viability was measured by MTT assay. The nature of the drug interaction was analyzed by using the combination index $(\mathrm{Cl})$ according to the method of Chou and Talalay. A Cl value lower than 0.90 indicates synergism; a $\mathrm{Cl}$ value between 0.90 and 1.10 indicates additive; and a $\mathrm{Cl}$ value higher than 1.10 indicates antagonism. Data analysis was performed by the Calcusyn software (Biosoft, Oxford, UK).

Mouse xenograft tumors study. For tumorigenesis assessment, the viable A549 or A549/T cells $\left(5 \times 10^{6} / 100 \mu\right.$ l PBS per mouse), as confirmed by trypan blue staining, were subcutaneously injected into the right flank of 7- to 8-week-old male SCID mice. After 28 days, the mice were killed and the tumors were excised and stored at $-80^{\circ} \mathrm{C}$ until western blotting. To determine the in vivo antitumor activity of SNOH-3 combined with paclitaxel (Taxol), viable A549/T cells $\left(5 \times 10^{6} / 100 \mu\right.$ PBS per mouse) were subcutaneously injected into the right flank of 7- to 8-week old male SCID mice. When the average tumor volume reached $50 \mathrm{~mm}^{3}$, the mice were randomly divided into four treatment groups, including control (saline only, $n=4$ ), SNOH-3 (40 mg/kg/3 days, i.p.; $n=4)$, Taxol ( $10 \mathrm{mg} / \mathrm{kg} /$ week, i.v.; $n=4)$, and the combination $(n=4)$. Tumor size was measured once every 3 days with a caliper (calculated volume $=$ shortest diameter ${ }^{2} \times$ longest diameter/2). Body weight was recorded once every 3 days. After 21 days, the mice were killed and the tumors were excised and stored at $-80^{\circ} \mathrm{C}$ until further analysis. These studies were performed in strict accordance with the recommendations in the Guide for the Care and Use of Laboratory Animals of the National Institutes of Health. The protocol was approved by the Committee on the Ethics of Animal Experiments of the Shenyang Pharmaceutical University.

Immunohistochemistry and TUNEL assay. Tissues embedded in paraffin were cut to a section of $4 \mu \mathrm{m}$, deparaffinized, and treated with citrate buffer. Then, they were blocked with avidin/biotin for $20 \mathrm{~min}$. The slides were incubated with anti-CD31 for overnight at $4^{\circ} \mathrm{C}$. Next, the slides were treated with secondary antibody with horseradish peroxidase goat anti-rabbit for $1-3 \mathrm{~h}$ and developed with 3,3-diaminobenzidine (Sigma-Aldrich, St. Louis, MO, USA). Finally, the slides were counterstained with hematoxylin. The microvessel density (MVD) was determined in a blinded manner by counting the total number of vessels in three fields. TUNEL system (Roche, Basel, Switzerland) was used to detect apoptosis in the tumor sections placed on slides according to the manufacturer's 
protocol. TUNEL reaction solution was substituted with TdT-free solution for a negative control. Sections were pretreated $10 \mathrm{~min}$ with DNase and visualized by DAB staining. Positive nuclei were identified by brown color. The percentage of positive cells over the total cells counted was calculated.

Statistical analysis. Differences between experimental groups were evaluated by one-way ANOVA or Turkey's post-hoc test using the SPSS11.5 software package for Windows (SPSS, Chicago, IL, USA). Survival curves were constructed using the Kaplan-Meier method. Statistical significance was based on a $P$-value of 0.05 $(P<0.05$, two-tailed test $)$

\section{Conflict of Interest}

The authors declare no conflict of interest.

Acknowledgements. We gratefully acknowledge financial support from the National High Technology Research and Development Program of China (863 Program) (No. 2012AA020305), National Key Scientific Project for New Drug Discovery and Development (2013ZX09301305), National Natural Science Foundation of China (No. 81572947), Program for Liaoning Innovative Research Team in University(LT2014023), Excellent Talents Plan in Liaoning Province (No: LJQ2014111), Shenyang Pharmaceutical University Science Foundation (No. ZCJJ2013401), Discipline Construction Program of Shenyang Pharmaceutical University (No. 52134606) and Natural Science Foundation of Hubei Province, China (No. 2015CFB280).

1. Torre LA, Bray F, Siegel RL, Ferlay J, Lortet-Tieulent J, Jemal A. Global cancer statistics, 2012. CA Cancer J Clin 2015; 65: 87-108.

2. Reck M, Heigener DF, Mok T, Soria JC, Rabe KF. Management of non-small-cell lung cancer: recent developments. Lancet 2013; 382: 709-719.

3. Evans T. Chemotherapy in advanced non-small cell lung cancer. Semin Respir Crit Care Med 2005; 26: 304-313.

4. Simon GR. Nab-Paclitaxel for the treatment of advanced squamous non-small-cell lung cancer: a comprehensive update. Clin Lung Cancer 2014; 15: 391-397.

5. Socinski MA. Update on taxanes in the first-line treatment of advanced non-small-cell lung cancer. Curr Oncol 2014; 21: e691-e703.

6. Joshi M, Liu X, Belani CP. Taxanes, past, present, and future impact on non-small cell lung cancer. Anticancer Drugs 2014; 25: 571-583.

7. Rosell R, Felip E. Predicting response to paclitaxel/carboplatin-based therapy in non-small cell lung cancer. Semin Oncol 2001; 28: 37-44.

8. Yeh JJ, Hsu WH, Wang JJ, Ho ST, Kao A. Predicting chemotherapy response to paclitaxelbased therapy in advanced non-small-cell lung cancer with P-glycoprotein expression. Respiration 2003; 70: 32-35.

9. Ajabnoor GM, Crook T, Coley HM. Paclitaxel resistance is associated with switch from apoptotic to autophagic cell death in MCF-7 breast cancer cells. Cell Death Dis 2012; 3: e260.

10. Le XF, Bast RC Jr. Src family kinases and paclitaxel sensitivity. Cancer Biol Ther 2011; 12 260-269.

11. Peng X, Gong F, Chen Y, Jiang Y, Liu J, Yu M et al. Autophagy promotes paclitaxel resistance of cervical cancer cells: involvement of Warburg effect activated hypoxia-induced factor 1- $\alpha$-mediated signaling. Cell Death Dis 2014; 5: e1367.

12. Brown R, Curry E, Magnani L, Wilhelm-Benartzi CS, Borley J. Poised epigenetic states and acquired drug resistance in cancer. Nat Rev Cancer 2014; 14: 747-753.

13. Minucci S, Pelicci PG. Histone deacetylase inhibitors and the promise of epigenetic (and more) treatments for cancer. Nat Rev Cancer 2006; 6: 38-51.

14. New M, Olzscha H, La Thangue NB. HDAC inhibitor-based therapies: can we interpret the code? Mol Oncol 2012; 6: 637-656.

15. Falkenberg KJ, Johnstone RW. Histone deacetylases and their inhibitors in cancer, neurological diseases and immune disorders. Nat Rev Drug Discov 2014; 13: 673-691.

16. West AC, Johnstone RW. New and emerging HDAC inhibitors for cancer treatment. J Clin Invest 2014; 124: 30-39.

17. Wang $L$, Chen $G$, Chen $K$, Ren $Y$, Li H, Jiang $X$ et al. Dual targeting of retinoid $X$ receptor and histone deacetylase with DW22 as a novel antitumor approach. Oncotarget 2015; 6: 9740-9755.
18. Perego P, Zuco V, Gatti L, Zunino F. Sensitization of tumor cells by targeting histone deacetylases. Biochem Pharmacol 2012; 83: 987-994.

19. Lin YC, Lin YC, Shih JY, Huang WJ, Chao SW, Chang YL et al. DUSP1 expression induced by HDAC1 inhibition mediates gefitinib sensitivity in non-small cell lung cancers. Clin Cancer Res 2015; 21: 428-438.

20. Raha P, Thomas S, Thurn KT, Park J, Munster PN. Combined histone deacetylase inhibition and tamoxifen induces apoptosis in tamoxifen-resistant breast cancer models, by reversing Bcl-2 overexpression. Breast Cancer Res 2015; $17: 26$.

21. Ramalingam SS, Maitland ML, Frankel P, Argiris AE, Koczywas M, Gitlitz B et al. Carboplatin and Paclitaxel in combination with either vorinostat or placebo for first-line therapy of advanced non-small-cell lung cancer. J Clin Oncol 2010; 28: 56-62.

22. Sachweh MC, Drummond CJ, Higgins M, Campbell J, Laín S. Incompatible effects of p53 and HDAC inhibition on p21 expression and cell cycle progression. Cell Death Dis 2013; 4: 0533.

23. Tewari M, Quan LT, O'Rourke K, Desnoyers S, Zeng Z, Beidler DR et al. Yama/CPP32 beta, a mammalian homolog of CED-3, is a CrmA-inhibitable protease that cleaves the death substrate poly(ADP-ribose) polymerase. Cell 1995; 81: 801-809.

24. Chung AS, Lee J, Ferrara N. Targeting the tumour vasculature: insights from physiological angiogenesis. Nat Rev Cancer 2010; 10: 505-514.

25. Huang Y, Chen J, Lu C, Han J, Wang G, Song C et al. HDAC1 and Klt4 interplay critically regulates human myeloid leukemia cell proliferation. Cell Death Dis 2014; 5: e1491.

26. Ray A, Alalem M, Ray BK. Loss of epigenetic Kruppel-like factor 4 histone deacetylase (KLF-4-HDAC)-mediated transcriptional suppression is crucial in increasing vascular endothelial growth factor (VEGF) expression in breast cancer. J Biol Chem 2013; 288: $27232-27242$.

27. Jamaladdin S, Kelly RD, O'Regan L, Dovey OM, Hodson GE, Millard CJ et al. Histone deacetylase (HDAC) 1 and 2 are essential for accurate cell division and the pluripotency of embryonic stem cells. Proc Natl Acad Sci USA 2014; 111: 9840-9845.

28. Dovey OM, Foster CT, Cowley SM. Histone deacetylase 1 (HDAC1), but not HDAC2, controls embryonic stem cell differentiation. Proc Natl Acad Sci USA 2010; 107: 8242-8247.

29. Xu Y, Jiang Z, Yin P, Li Q, Liu J. Role for Class I histone deacetylases in multidrug resistance. Exp Cell Res 2012; 318: 177-186.

30. Keshelava N, Davicioni E, Wan Z, Ji L, Sposto R, Triche TJ et al. Histone deacetylase 1 gene expression and sensitization of multidrug-resistant neuroblastoma cell lines to cytotoxic agents by depsipeptide. J Natl Cancer Inst 2007; 99: 1107-1119.

31. Shah RD, Jagtap JC, Mruthyunjaya S, Shelke GV, Pujari R, Das G et al. Sodium valproate potentiates staurosporine-induced apoptosis in neuroblastoma cells via Akt/survivin independently of HDAC inhibition. J Cell Biochem 2013; 114: 854-863.

32. Rosato RR, Almenara JA, Grant S. The histone deacetylase inhibitor MS-275 promotes differentiation or apoptosis in human leukemia cells through a process regulated by generation of reactive oxygen species and induction of p21CIP1/WAF1. Cancer Res 2003; 63: $3637-3645$.

33. Dowdy SC, Jiang S, Zhou XC, Hou X, Jin F, Podratz KC et al. Histone deacetylase inhibitors and paclitaxel cause synergistic effects on apoptosis and microtubule stabilization in papillary serous endometrial cancer cells. Mol Cancer Ther 2006; 5: 2767-2776.

34. Kaewpiboon C, Srisuttee R, Malilas W, Moon J, Oh S, Jeong HG et al. Upregulation of Stat1HDAC4 confers resistance to etoposide through enhanced multidrug resistance 1 expression in human A549 lung cancer cells. Mol Med Rep 2015; 11: 2315-2321.

35. Hutt DM, Roth DM, Vignaud H, Cullin C, Bouchecareilh M. The histone deacetylase inhibitor, Vorinostat, represses hypoxia inducible factor 1 alpha expression through translational inhibition. PLOS One 2014; 9: e106224.

36. Kong X, Lin Z, Liang D, Fath D, Sang N, Caro J. Histone deacetylase inhibitors induce VHL and ubiquitin-independent proteasomal degradation of hypoxia-inducible factor 1 alpha. Mol Cell Biol 2006; 26: 2019-2028.

Cell Death and Disease is an open-access journal published by Nature Publishing Group. This work is licensed under a Creative Commons Attribution 4.0 International License. The images or other third party material in this article are included in the article's Creative Commons license, unless indicated otherwise in the credit line; if the material is not included under the Creative Commons license, users will need to obtain permission from the license holder to reproduce the material. To view a copy of this license, visit http://creativecommons.org/licenses/by/4.0/ 